\title{
Presencia de las redes sociales en las campañas publicitarias transmedia más premiadas
}

\author{
José MARTínez SÁEZ \\ Universidad CEU Cardenal Herrera \\ pemartinez@uch.ceu.es \\ José Manuel Amiguet Esteban \\ Universidad CEU Cardenal Herrera \\ jamiguet@uch.ceu.es \\ Rosa Visiedo Claverol \\ Universidad CEU Cardenal Herrera \\ rvisiedo@uch.ceu.es
}

\begin{abstract}
Resumen:
El objetivo del presente artículo es determinar en qué medida las campañas publicitarias que pueden ser tipificadas como transmedia incorporan el uso de redes sociales, qué tipos de redes sociales y con qué tipo de interacción. En primer término identificaremos la presencia de la transmedialidad en las campañas más premiadas en 2012. Y a continuación analizaremos la presencia de redes sociales en la estructura de las campañas que sean transmedia atendiendo no sólo al criterio cuantitativo sino también desde una perspectiva cualitativa (en qué tipo de redes y con qué grado de interacción).
\end{abstract}

Palabras clave: publicidad; redes sociales; transmedia; internet; digital.

\section{Presence of social networks in the most awarded transmedia advertising campaigns}

\begin{abstract}
:
The aim of this research is to determine to what extent advertising campaigns that can be classified as transmedia incorporate the use of social media, what types of social media and with what kind of interaction. First we will identify the presence of transmediality in the most awarded campaigns in 2012. And then we will analyze the presence of social media in the structure of transmedia campaigns taking into account not only quantitative criteria but also from a qualitative perspective (what kind of social networks and with what degree of interaction).
\end{abstract}

Key Words: advertising; social media; transmedia; internet; digital.

\section{Referencia normalizada:}

Martínez Sáez, J., Amiguet Esteban, J. M. y Visiedo Claverol, R. (2014): Presencia de las redes sociales en las campañas publicitarias transmedia más premiadas. Historia y Comunicación Social. Vol. 19. Núm. Especial Marzo. Págs. 301-313.

Sumario: 1.- Introducción; 2.- Marco teórico; 3.- Objetivos y metodología; 4.- Resultados; 5.- Conclusiones; 6.- Referencias Bibliográficas 


\section{Introducción}

En la actualidad en el entorno publicitario se asiste a un momento de transición, a un cambio de paradigma ocasionado o acentuado en buena medida por el impacto de lo digital.

Hacemos la precisión a ese carácter de acelerador de la tecnología digital puesto que no puede señalarse como el único origen de la actual desorientación del sector publicitario. Si rebobinamos en el tiempo podemos observar como Joan Costa ya apuntaba a una crisis del sistema publicitario y propugnaba una nueva concepción del negocio bajo la óptica de un ecosistema de la comunicación (Costa, J., 1992: 58). Aquel ecosistema pretendía trascender la mirada corta sobre la publicidad como técnica y que la gestión de la comunicación de las organizaciones se abordara de manera holística. Indudablemente hay en la actualidad una mayor conciencia de que todo comunica en una organización pero estamos lejos de ese ecosistema ideal que proponía Costa y eso que cuando él lo esbozó no tuvo en cuenta el impacto de la comunicación digital.

Lejos estamos de ese ecosistema de Costa. Muy lejos. Y más si pensamos que cuando Costa dibujó su esquema en 1992 no tenía en cuenta la entonces incipiente comunicación interactiva. Lo cierto es que sigue habiendo desencuentros entre la gestión de la comunicación comercial de las marcas y la comunicación corporativa de las compañías que las nutre.

En ese sentido podemos encontrar cierto paralelismo en el desencuentro que se da entre la publicidad masiva tradicional y la publicidad online. Entre las agencias mainstream y las nuevas agencias digitales ha habido desde mediados de los noventa una relación plena de suspicacias y susceptibilidades (McStay, A., 2010:29). De hecho la dificultad de integrar lo digital es la causa de que existieran pequeñas estructuras de especialistas que vinieron a denominarse "agencias digitales" que con menos recursos que los dedicados a los medios convencionales lograban notorias acciones de comunicación que incluso podían nacer en on-line y de ahí con su éxito saltar a los medios masivos (Ibid.:30). Afortunadamente parece que las diferencias se borran y atendemos ahora a una voluntad clara de aproximarse a la comunicación con intención media neutral proponiendo contenidos que los usuarios pueden seguir a través de distintos medios, tanto offline como online. Contenidos que pueden nacer como un espot convencional para acabar formando parte de una conversación con los usuarios a través de Twitter y Youtube, como en el caso de la exitosa campaña de 2010 de Old Spice. O también se puede abrir un concepto de comunicación con un espot y un corto documental y a partir de ahí enriquecer y hacer crecer la conversación a través de juegos, websites y acciones de street marketing como en el caso de Coca Cola con su Happiness factory. Es la presencia de lo Transmedia (JWT Intelligence: 2011).

En ambos casos, en el de Old Spice y en el de Cocacola, Internet tenía una presencia casi nuclear. Y hoy un elemento clave de Internet son las redes sociales que han acabado por afectar de manera radical a los modos de ocio y relación de los consu- 
midores. De hecho las redes sociales han transformado el modo de consumir los productos culturales en televisión dando lugar a la Social TV.

En el presente artículo trataremos de dar cuenta de cómo el concepto transmedia se aplica a la actividad publicitaria y cuál es el rol de los medios sociales en la interrelación de las marcas con sus públicos en la actualidad.

\section{Marco teórico}

La comunicación de masas comenzó a estudiarse en la segunda década del siglo XX desde diferentes paradigmas de lo más variados: el informacional de Shannonn y Weaver, el crítico de la Escuela de Francfort, el empirico-analítico de Lasswell, Lazarsfeld, Merton, Berelson..., el ecléctico de los Cultural Studies y las diferentes aproximaciones semiótico-discursivas de Saussure, Peirce, Barthes, Metz, Eco... (Scolari, C., 2009: 33-43). La mayoría de teorías sustentadas al amparo de estos paradigmas no son capaces de dar cuenta de la comunicación en la era digital.

Con lo digital hemos asistido a un cambio de modelo. Del Modelo Difusión, propio de los medios de comunicación de masas, cuyo medio paradigmatico es la televisión, pasamos al Modelo Reticular que posibilita la tecnología digital (Martínez, J. y Palao, J.A., 2009). En el Modelo Reticular se dan una serie de características como la hipertextualidad, la interactividad, lo virtual, la conectividad, la multimedialidad y la modularidad que exigen una revisión a fondo de su marco teórico. Algunas aproximaciones se han efectuado y de ellas queremos dar una ligera semblanza.

Internet ha afectado seriamente a la forma en que consumimos productos culturales y en ese sentido Himanen (2001) teoriza el informacionalismo y lo define principalmente como "un paradigma tecnológico". Tanto Himanen como Lev Manovich (2006:82) identifican en las nuevas tecnologías las características clave que inspiran el cambio de paradigma: la capacidad de recombinar la información y la flexibilidad distributiva. Ese cambio de paradigma puede ser observado desde una perspectiva liberadora como una suerte de "comunismo epistémico" puesto que Pekka Himanen atribuye al fenómeno Internet la capacidad de generar una nueva ética: la ética hacker, una ética que apuesta por compartir de manera abierta y desinteresada la información como algo opuesto a la ética del capitalismo (2001:11). Una de las consecuencias más interesantes e inquietantes es la quiebra del concepto de autor. No es sólo una cuestión cultural o estética, sino dicha ética en virtud de la piratería informática afecta radicalmente a toda la estructura del negocio de la producción, distribución y exhibición de productos culturales y de entretenimiento. Anderson (2009) lo explica de manera diferente. Para él nos encontramos en la transición de diferentes sistemas económicos. Estamos pasando de la economía del átomo a la economía del bit. Lo propio de la economía del átomo es la lógica de la inflación. Pero el rasgo característico de la economía del bit, señala Anderson, es la lógica deflacionaria con tendencia a coste cero. El usuario, en consonancia con el informacionalismo de Himanen, no 
está dispuesto a pagar por acceder a los contenidos. Entra en crisis el sistema de los medios y con él todo el sistema publicitario convencional. Es decir, cuando los medios más necesidad tienen de publicidad porque el público no quiere pagar por los contenidos, la publicidad menos necesita a los medios convencionales (McStay, A., 2010:23).

Otra aproximación es la de De Kerckhove. Éste apunta a una nueva mutación al distinguir entre el hombre-masa, asociado al Modelo Difusión de la televisión y el hombre-velocidad propio del Modelo Reticular. En el Modelo Difusión, la televisión por ejemplo es autónoma en su flujo, respecto a los impulsos del espectador, pero en el Modelo Reticular es el hombre-velocidad el que se encuentra en todas partes, en el centro de las cosas. El hombre-velocidad no es principalmente consumidor sino que potencialmente es productor y agente (de Kerchove, D. 1999:160).

La Social TV es un punto de encuentro entre el Modelo Difusión y el Modelo Reticular. Se habla de Social TV cuando los espectadores emplean las redes sociales para prolongar la relación con los relatos o programas que siguen en televisión. Los smart-phones y tablets devienen en segundas pantallas (Proulx, M. y Shepatin, S., 2012: 83-109). "Segundas pantallas" amplificadoras del impacto de la televisión más allá de su momento en la programación y que se convierten además en una oportunidad para las marcas.

Los conceptos críticos del nuevo paradigma son el hipertexto y la interactividad. El hipertexto posibilita que la lectura no siga un orden prefijado sino que sea el lector el que pueda decidir entre diferentes nexos de unión siendo capaz e llegar a documentos originales a través de breves citas. En la visión de Ted Nelson que glosa Berners-Lee, en su Xanadú, cada cita tendría un vínculo que la devolvería a su fuente, permitiendo a los autores originales ser compensados con una pequeña cantidad cada vez que se leyese la cita. Es la quimera de una sociedad utópica en la que toda la información pudiera ser compartida (Ted Nelson en Berners-Lee, T., 2000:5). Ya hemos hablado antes con Himanen de la quiebra de la noción de autor, quiebra que queda acentuada por la accesibilidad generalizada y la independencia de la secuencialidad gracias al hipertexto. Con la interactividad se da lugar a una concepción múltiple de la autoría (Manovich, L., 2006:82). O en palabras de Andrew Darley la interactividad da paso a un autor descentrado (2002:215-218).

La interactividad implica una suspensión del acto clásico de la lectura con importantes consecuencias en la creación de contenidos culturales y publicitarios. La interactividad supone, entre otras cosas, la posibilidad de modificar el transcurso de los relatos. En ese sentido, con el hipertexto el sujeto no ejerce de argamasa de la coherencia textual. Por eso el recurso al simulacro, el énfasis en la experiencia y el retorno al puro espectáculo y a lo sensitivo tanto en el entretenimiento digital como en la publicidad digital.

Aquí podemos encontrar al tiempo el auge de la transmedialidad: los usuarios fluyen a través de diferentes medios siguiendo los contenidos e interactuando con ellos. Además, como señala Jeff Gómez, en una producción transmedia el usuario 
puede participar de la historia y convertirse en co-creador de la misma a través de los medios sociales o las aplicaciones móviles (en Scolari, C. 2013: 42). Jenkins (2008) afirma el papel centrípeto del hombre-velocidad kerchoviano cuando afirma que en la era transmedia la audiencia abandona el rol de espectador y asume un papel más participativo.

Por lo tanto, las narrativas transmedia responden a estrategias que aprovechan la existencia y convergencia de formatos y plataformas para elaborar y distribuir mensajes. Lo transmedia supone la elaboración de mensajes complejos aprovechando las cualidades de cada soporte para la configuración de un mensaje global constituido a través de mensajes independientes. Es decir, una producción transmedia no se limita a ser un relato adaptado a diferentes medios sino que un único universo narrativo se desarrolla y fluye a través de un conjunto de medios. Cada medio y cada producción individual debe tener sentido por sí misma al tiempo que pertenece a un mundo diegetico común en el que la participación de los usuarios es clave.

Podemos decir que el concepto transmedia es elástico y fluido. $\mathrm{O}$ siguiendo a Zgyman Bauman (2003) es "líquido". De hecho Jenkins habla de medios y contenidos "esparcibles" (Jenkins et al., 2013). Spreadable en el inglés original. También Manovich y Daniel Solana utilizan la analogía con lo líquido para caracterizar los nuevos medios.

En el momento crítico que vive la publicidad convencional el concepto transmedia ofrece nuevas formas de contar historias, y puede ser utilizado por las marcas para crear un contenido más atractivo y experiencial.

\section{Objetivos y metodología}

Los objetivos de la presente investigación necesitan de un objetivo previo: la determinación de cuál es la presencia de la transmedialidad en las campañas publicitarias mejor consideradas desde la percepción de los profesionales de la creatividad publicitaria. Y así subsidiariamente llegar a descubrir en qué medida las campañas publicitarias transmedia incorporan el uso de redes sociales, qué tipos de redes sociales y con qué tipo de interacción.

Uno de los rasgos característicos de las narraciones transmedia es la participación del usuario en la circulación de los contenidos o, incluso, en la co-creación de los mismos. Esto es, no solo supone que las historias fluyen narrativamente a través de diferentes plataformas, sino que también implica la participación del usuario en los contenidos como rasgo característico. Y esta participación del usuario se da en muchas ocasiones en redes sociales.

Nuestra hipótesis es que las campañas que pueden ser catalogadas como transmedia incorporan las redes sociales como canal de interacción. 
Por eso, en nuestro trabajo comenzaremos por identificar en primer término la presencia de la transmedialidad en las campañas más premiadas en 2012. Y a continuación analizaremos la presencia de redes sociales en la estructura de esas campañas transmedia atendiendo no sólo al criterio cuantitativo sino también desde una perspectiva cualitativa (en qué tipo de redes y con qué grado de interacción).

El análisis que da pie a los resultados se centra en un corpus extraído de los grandes premios y los "oros" de los palmarés de los principales festivales publicitarios en 2012 en las categorías de campañas integradas y/o interactivas.

Desde el punto de vista del método, en nuestro trabajo el objetivo de la investigación es la observación de un fenómeno: la incorporación de lo transmedia a la producción publicitaria y cómo en esas campañas transmedia obtienen protagonismo las redes sociales En línea con el pluralismo metodológico propio de las Ciencias Sociales y a la definición de Beltrán de las vías de acceso a la realidad social (1996:32), nuestro método será cuantitativo por cuanto queremos medir cuántas campañas son transmedia, si usan redes sociales y qué tipos de redes sociales, pero también cualitativo puesto que nuestro objetivo no es solo cuantificar sino que además buscamos la identificación de los tipos de interacción de los usuarios.

Respecto al diseño de la investigación hemos acudido al Análisis de Contenido Adhoc como técnica. Ya que siendo cuantitativa, pues identifica y cuantifica categorías, ofrece también a través de una adecuada categorización una aproximación cualitativa al fenómeno estudiado. El análisis de contenido es "una técnica de investigación para la descripción objetiva, sistemática y cuantitativa del contenido manifiesto de las comunicaciones, con el fin de interpretarlas" (Berelson en Krippendorff, K., 1990:29). En definitiva, la técnica es cuantitativa en su proceso pero tiene un fin de interpretación del fenómeno estudiado lo que le confiere el cariz cualitativo.

Partiendo de esta concepción de la técnica del Analisis de Contenido efectuamos el mismo con las siguientes selecciones previas extraídas de Pîñuel (2002:7):

- Determinación de la comunicación estudiada: las campañas de publicidad consideradas más creativas.

- Selección de las categorías a emplear en el análisis: valor transmedia, uso de redes sociales, nivel de interacción.

- Unidades de análisis: medios empleados en las campañas, redes sociales usadas, tipo de interacción con el usuario.

- Sistema de recuento y medida: visionado de los case study en video y búsqueda heurística en perfiles sociales.

El universo lo constituyen las campañas mejor consideradas por los propios creativos. Para ello hemos acudido a una selección de las campañas premiadas en festivales publicitarios nacionales e internacionales, ya que en los festivales los jurados se constituyen principalmente por creativos de las agencias. Para el corpus de la investigación hemos extraído los grandes premios y los oros de las categorías de 
campañas integradas y/o digitales (o con sus nomenclaturas similares) en los siguientes festivales:

- Cannes

- Cresta

- Clio

- $\quad$ Effie

- D\&AD

- $\quad$ FIAP

- $\quad$ El Sol

La selección tras la lógica depuración de duplicidades (ya que hay campañas premiadas en más de un festival) ha dado con un corpus de 50 campañas. Sobre ellas realizamos el Análisis de Contenido Ad hoc.

\section{Resultados}

Las dos cualidades esenciales que permiten la categorización como transmedia (Scolari, C., 2013:45) son: 1) que el contenido se expanda a través de diferentes canales (Jeff Gómez señala que un contenido transmedia debe fluir a través de 3 plataformas distintas o más); y 2) que el usuario colabore en ese proceso expansivo. O sea, que el usuario interactúe con el contenido en mayor o menor grado.

Tras el análisis del corpus detectamos la presencia de transmedialidad en el $66 \%$ de las campañas premiadas. En concreto, en 33 de las 50 campañas analizadas:

Tabla 1. Presencia de la variable Transmedia

\begin{tabular}{|l|l|l|}
\hline & \multicolumn{1}{|c|}{ campaña } & \multicolumn{1}{c|}{ marca } \\
\hline 1 & All is not lost & Google \\
\hline 2 & Conecting Lifelines & Honda \\
\hline 3 & Obsessed whit sound & Philips \\
\hline 4 & A day with nike - Fuel Band & Nike \\
\hline 5 & Day one & Prudential \\
\hline 6 & Ted Williams fight hunger & Kraft \\
\hline 7 & Rivers os Light & Gobierno de Colombia \\
\hline 8 & VIRTUAL 2PAC & Coachella \\
\hline 9 & Just Dance 3 & Ubisoft \\
\hline 10 & Star Player & Heineken \\
\hline 11 & NYC - Rescue Mission// Underheard in NY & NYC Rescue Mission \\
\hline 12 & Sneakerpedia & Footlocker \\
\hline 13 & Sparq Code & SPARQCODE + NIKE \\
\hline
\end{tabular}




\begin{tabular}{|l|l|l|}
\hline 14 & Hot Wheels for Real & Hot Wheels - Mattel \\
\hline 15 & Romanians & ROM \\
\hline 16 & Vanish Australia & Vanish \\
\hline 17 & Battle fot the Babies & B92 Foundation \\
\hline 18 & The Face of Marathon & Adidas \\
\hline 19 & CNN Ecosphere project \#cop17 & CNN \\
\hline 20 & Living Young Experience & Evian \\
\hline 21 & Feesding Tube & truth \\
\hline 22 & Awomansnightmare & samusocial \\
\hline 23 & Kaiser Chief - The medieval & Kaiser Chief - Universal \\
\hline 24 & Shop Small Saturday & Shop Small - American \\
\hline 25 & Book Burning Party & Express \\
\hline 26 & This Mom Hates This & Troy Library \\
\hline 27 & The Love history & Dead Space 2 (EA) \\
\hline 28 & Decode Jay-Z & Chobani \\
\hline 29 & Search For The World Championship Baby & Bing \\
\hline 30 & KID Rescue & Funcast \\
\hline 31 & Coversaciones & Banco Sabadell Telefónica \\
\hline 32 & Experimento comparte & Acción contra el hambre \\
\hline 33 & Arriba Contador & FLEX \\
\hline
\end{tabular}

El siguiente paso fue identificar el uso de redes sociales en las campañas tipificadas como transmedia. Como hemos visto, la participación del usuario es necesaria para poder catalogar una campaña como transmedia.

Tabla 2. Uso de Redes Sociales

\begin{tabular}{|c|c|c|c|c|}
\hline marca & facebook & twitter & youtube & otras \\
\hline Honda & $\mathrm{Si}$ & $\mathrm{Si}$ & $\mathrm{Si}$ & Ustream \\
\hline Philips & SI & SI & SI & Linkedin \\
\hline Nike & SI & & SI & \\
\hline Prudential & SI & & & \\
\hline Kraft & & & SI & \\
\hline Coachella & & & SI & \\
\hline Ubisoft & $\mathrm{Si}$ & & SI & Vimeo \\
\hline Heineken & SI & SI & & \\
\hline NYC Rescue Mission & & SI & SI & \\
\hline Footlocker & SI & SI & & MySpace \\
\hline SPARQCODE + NIKE & & & SI & \\
\hline Hot Wheels - Mattel & SI & & SI & \\
\hline ROM & SI & & SI & \\
\hline Vanish & SI & SI & & \\
\hline B92 Foundation & SI & & SI & \\
\hline $\mathrm{CNN}$ & SI & SI & SI & \\
\hline Evian & SI & & SI & \\
\hline
\end{tabular}




\begin{tabular}{|l|l|l|l|l|}
\hline truth & Si & SI & SI & \\
\hline samusocial & SI & SI & & \\
\hline $\begin{array}{l}\text { Kaiser Chief - } \\
\text { Universal }\end{array}$ & SI & SI & & \\
\hline $\begin{array}{l}\text { Shop Small - American } \\
\text { Express }\end{array}$ & SI & SI & Si & $\begin{array}{l}\text { Foursquare; Google } \\
\text { Map }\end{array}$ \\
\hline Troy Library & SI & SI & SI & Foursquare, eBay... \\
\hline Dead Space 2 (EA) & SI & SI & SI & \\
\hline Chobani & SI & SI & SI & \\
\hline Bing & SI & SI & & \\
\hline Comcast & Si & SI & & \\
\hline Banco Sabadell & & & SI & \\
\hline $\begin{array}{l}\text { Acción contra el } \\
\text { hambre }\end{array}$ & & & SI & \\
\hline FLEX & SI & \multicolumn{2}{|l|}{} \\
\cline { 2 - 5 } & 23 & 15 & SI & Tuenti \\
\cline { 2 - 4 } & & \multicolumn{2}{|l}{} &
\end{tabular}

Las redes sociales estaban en el $88 \%$ de las campañas consideradas transmedia. En concreto en 29 campañas. Respecto al total del corpus la presencia conjunta de transmedia y redes sociales se da en el 58\% de las campañas. La red social más empleada es Facebook en 23 ocasiones. A continuación Youtube (en 21 de las campañas analizadas) y en tercer lugar Twitter. El uso de otras redes sociales como Google maps, Linkedin, Foursquare, Myspace o el caso español de Tuenti es marginal.

Finalmente, nos interesaba evaluar el tipo de participación del usuario. Establecimos 3 categorías con un nivel de implicación del usuario progresivo:

- Difusión. Cuando la participación del usuario oscila entre limitarse los "me gusta" o convertirse en pieza clave de la campaña contribuyendo a la difusión de manera activa y viralizando el contenido.

- Conversación. En esta categoría incluimos exclusivamente aquellas campañas que, de manera explícita y evidente, implican a los usuarios para que conversen entre ellos a través de distintas plataformas digitales o de redes sociales. Generalmente son ejemplos de Social TV. Es decir, categorizamos como conversación cuando ésta, más allá del deseable eco social de cualquier campaña, forma parte intrínseca de la misma.

- Co-creación. Cuando el usuario actúa y crea o modifica el contenido de la campaña en el sentido deseado por los creativos. Es la categoría donde el usuario debe realizar mayor esfuerzo, colgando fotos, vídeos o manipulando fotos y vídeos, por ejemplo. Es lo que se conoce como UGC (User Generated Content)

El resultado es que la participación de los usuarios en las campañas más premiadas oscilaba entre los dos polos de la posible implicación. De las 29 campañas mientras 
en 14 se daba únicamente la difusión en otras 12 había co-creación por parte del usuario.

Tabla 3. Tipo de participación del usuario

\begin{tabular}{|l|l|}
\hline marca & \\
\hline Honda & difusión \\
\hline Philips & co-creación \\
\hline Nike & difusion \\
\hline Prudential & co-creación \\
\hline Kraft & difusión \\
\hline Coachella & difusión \\
\hline Ubisoft & co-creación \\
\hline Heineken & conversacion \\
\hline Underheard & difusión \\
\hline Footlocker & co-creación \\
\hline SPARQCODE + NIKE & co-creación \\
\hline Hot Wheels - Mattel & difusión \\
\hline ROM & co-creación \\
\hline Vanish & conversacion \\
\hline Battle fot the Babies & difusión \\
\hline CNN & conversacion \\
\hline Evian & co-creación \\
\hline truth & difusión \\
\hline samusocial & difusión \\
\hline Kaiser Chief - Universal & co-creación \\
\hline Shop Small - American Express & difusion \\
\hline Library & conversacion \\
\hline Dead Space 2 (EA) & difusión \\
\hline Chobani & co-creación \\
\hline Bing & co-creación \\
\hline Comcast & co-creación \\
\hline Banco Sabadell & difusion \\
\hline Acción contra el hambre & difusion \\
\hline FLEX & difusion \\
\hline & \\
\hline
\end{tabular}

\section{Conclusiones}

Definitivamente podemos afirmar que según los resultados la variable transmedia está muy valorada por los creativos publicitarios, ya que ellos son los componentes de los jurados y, por tanto, son los responsables de los premios que han conformado el corpus de la investigación. Porque más del 65\% de las campañas analizadas podían ser conceptualizadas o tipificadas como transmedia. 
También podemos afirmar claramente que la mayoría de estas campañas usa las redes sociales para su relación con los usuarios. Ya que uno de los rasgos esenciales del transmedia es la participación de los usuarios y ésta se ha dado en las campañas analizadas en el $88 \%$ de los casos mediante redes sociales. Las redes sociales más utilizadas han sido en este orden facebook, youtube y twitter.

Por último y por lo que respecta al tipo de interacción que se da con los usuarios, en las campañas transmedia podemos observar como la presencia de la simple difusión queda destinada a aproximadamente la mitad de estas campañas, la otra mitad incluye una participación más activa de los usuarios bien a través de la conversación (solo en 3 casos), bien a través de la co-creación de contenidos.

También nos parece importante señalar que en ninguno de los casos estudiados había publicidad en facebook o en cualquiera de las otras redes sociales. El uso de los medios era para canalizar la relación con los usuarios pero siempre desde la motivación a la participación voluntaria. Lo cual da que pensar respecto al uso puramente publicitario de las redes sociales. Quizás acudamos a este aspecto en otra futura investigación.

\section{Bibliografía}

ANDERSON, Chris (2009): Gratis. El futuro de un precio radical. Ediciones Urano. Barcelona.

BARDIN, Laurence (2002): Análisis de contenido. Ediciones Akal. Madrid.

BELTRÁN, M. (1996): "Cinco vías de acceso a la realidad social”, en (Comp.) García Ferrando, M., Ibáñez, J. y Alvira, F.: El análisis de la realidad social. Métodos y técnicas de investigación. Alianza Editorial. Madrid.

BERNERS-LEE, Tim (2001): Tejiendo la red. Siglo XXI. Madrid.

COSTA, Joan (1992): Reinventar la publicidad. Reflexiones desde las Ciencias Sociales. Claves de Comunicación, Fundesco. Madrid.

DARLEY, Andrew (2002): Cultura Visual Digital. Espectáculo y nuevos géneros en los medios de comunicación. Paidós. Barcelona.

HIMANEN, Peka (2001): La ética del hacker y el espíritu de la información. Destino. Barcelona.

JENKINS, Henry (2008): Convergente culture. La cultura de la convergencia de los medios de comunicación. Paidós. Barcelona.

JENKINS, Henry (2013): Spreadable Media. Creating valuen and meaning in a networked culture. New York University Press. New York.

JWT Intelligence (2011): Transmedia Rising. Disponible en www.jwtintelligence.com/ production/TransmediaRising_JWT_TrendReport_March2011.pdf. [Consultado el 4 de marzo de 2011].

KERCKHOVE, Derrick de. (1999): La Piel de la cultura. Investigando la nueva realidad electrónica. Gedisa. Barcelona. 
KRIPPENDORFF, Klaus (1990): Metodología de análisis de contenido. Teoría y práctica. Paidós. Barcelona.

MANOVICH, Lev (2006): El lenguaje de los nuevos medios de comunicación. La imagen en la era digital. Paidós Comunicación. Barcelona.

MARTINEZ, J. y PALAO, J.A. (2009): El cine y el entorno visual. Material docente Módulo La innovación en la obra audiovisual. Valencia Internacional University. Disponible en www.viu.es. [Consultado el 27 de septiembre de 2010].

MCSTAY, Andrew, (2010): Digital Advertising. Palgrave Macmillan. London.

PIÑUEL, José Luis (2002): Epistemología, metodología y técnicas de análisis de contenido. Universidad Complutense de Madrid. Madrid.

PROULX, Mike y SHEPATIN, Stacey (2012): Social TV: how marketers can reach and engage audiences by connecting televisión to the web, social media and mobile. John Wiley\&Sons. New Jersey.

SCOLARI, Carlos (2008): Hipermediaciones. Elementos para una Teoría de la Comunicación Digital Interactiva. Gedisa. Barcelona.

SCOLARI, Carlos (2013): Narrativas Transmedia. Cuando todos los medios cuentan. Deusto. Barcelona.

SIERRA BRAVO, Restituto (2001): Técnicas de investigación social. Teoría y ejercicios. Paraninfo. Madrid.

SPURGEON, Christina (2008): Advertising and new media. Routledge. London.

WEBER, Larry (2009): Marketing to the Social Web. John Wiley\&Sons. New Jersey.

\section{Los autores}

José Martínez Sáez. Doctor en Comunicación. Profesor de Creatividad Publicitaria y Vicedecano de Pubicidad y Relaciones Públicas. Ha participado en diferentes proyectos europeos y ha sido investigador invitado en la Solent Southampton University en Reino Unido y en el Digital World Center en la Inholland de Rótterdam y profesor invitado en universidades belgas. El interés por el cambio de paradigma en el sistema publicitario le ha llevado a realizar distintas estancias en el extranjero en 2010 que han dado como resultado la realización de varias comunicaciones a congresos internacionales sobre el impacto digital en el sistema publicitario.

José Manuel Amiguet Esteban. Doctor en Ciencias de la Información por la Universidad Complutense. Es experto en la gestión del valor añadido en la marca. Es profesor de Creatividad Publicitaria. Ha realizado distintos trabajos presentados en congresos en torno a nuevas fórmulas de creatividad publicitaria como el advertainment y ha liderado otros proyectos de investigación como los que trataban de campañas de publicidad social en Marruecos o Senegal. Actualmente es Secretario General de la Universidad CEU Cardenal Herrera 
Rosa Visiedo Claverol. Doctora en Ciencias de la Información. Es Rectora de la Universidad CEU Cardenal Herrera en la actualidad. Además de su experiencia profesional posee una dilatada carrera académica. Es miembro de varios comités científicos y organizadores de congresos internacionales. Realizó su tesis doctoral sobre las estructuras de las agencias de publicidad en la Comunidad Valenciana y lo hizo empleando como técnica las entrevistas en profundidad. Sus últimas publicaciones versan sobre la comunicación política en la era digital. 